\title{
Comparación de la severidad de invasión asociada a caminos con diferentes niveles de perturbación en un bosque templado lluvioso del sur de Chile
}

\section{Comparison of the severity of invasion associated to pathways with different disturbance levels in a temperate rainforest in southern Chile}

\author{
Daniela Mardones ${ }^{1 *}$, Nicol Fuentes ${ }^{2} \&$ Alfredo Saldaña ${ }^{1}$ \\ 1Departamento de Botánica, Facultad de Ciencias Naturales y Oceanográficas, Universidad de Concepción, Casilla 160-C, \\ Concepción, Chile. \\ ${ }^{2}$ Laboratorio de Invasiones Biológicas, Facultad de Ciencias Forestales, Universidad de Concepción, Casilla 160-C, \\ Concepción, Chile. \\ *dmardonesagurto@gmail.com
}

\begin{abstract}
RESUMEN
La introducción de especies en áreas protegidas es un fenómeno promovido principalmente por actividad del hombre. Esto ha incrementado la preocupación por implementar métodos de evaluación de riesgo de invasión ('Weed Risk Assessment': WRA) y estimar cuán invadido se encuentra un lugar (severidad de invasión, SI). Además, la diversidad de una comunidad podría regular la introducción de especies, lo cual es dependiente de la escala. A escala regional, la relación entre la riqueza de plantas nativas e introducidas es frecuentemente positiva, pues existe mayor heterogeneidad ambiental que permite el desarrollo de ambos tipos de especies. A escala local la relación sería negativa, debido a que la competencia interespecífica sería inevitable. El Parque Nacional Puyehue corresponde a un ecosistema de bosque templado lluvioso que posee 55 especies introducidas. En este se evaluó qué especies introducidas tienen mayor potencial invasor (mayor WRA) y mayor éxito de invasión (mayor cobertura), y si existe una relación lineal positiva entre estos dos parámetros. También comparamos la SI en sitios con diferente intensidad de perturbación, y evaluamos la relación entre la diversidad de especies nativas e introducidas a escala local a orillas de camino. Aplicando métodos de evaluación de riesgo, encontramos que todas las especies introducidas en borde de camino presentaron alto potencial invasor, y además las que presentaron mayores puntajes de WRA tuvieron mayor cobertura. La SI fue mayor en sitios más perturbados, y no se encontró relación entre especies nativas e introducidas entre sitios con diferente intensidad de perturbación asociados a bordes de caminos. Proponemos tener en consideración a las especies con mayor potencial invasor en el parque para planes de mitigación, y además que el WRA es un método que podría ser aplicado para realizar evaluaciones de riesgo en áreas protegidas. Finalmente, destacamos el rol de los caminos como principales fuentes de propagación de las especies introducidas, aumentando la severidad de invasión de un determinado sitio.
\end{abstract}

Palabras clave: Bosque templado lluvioso, diversidad y abundancia, ecología de invasiones, plantas introducidas, WRA.

\begin{abstract}
Alien species introduction in protected areas is a phenomenon promoted primarily by human activity. This fact has increased the concern for implementing risk assessment methods to assess the species that may potentially become invasive ('Weed Risk Assessment', WRA), and estimate how invaded is a site (severity of invasion, SI). Moreover, the diversity of a community could regulate the introduction of species, which is scale dependent. At regional scale the relationship between both native and alien plant species is often positive, because greater environmental heterogeneity allows the development of this type of species. At a local scale, the relationship between native and alien diversity might be negative, because the competition would be inevitable. The Puyehue National Park corresponds to a temperate rainforest ecosystem that present 55 alien plant species. In this protected area we determined alien species that show both a high invasive potential (high WRA) and invasion success (high coverage). We also assessed whether there is positive relationship between these two parameters. We also compare the SI of sites that differ in disturbance intensity and evaluate the relationship between the diversity of native and introduced species at local level. Applying risk assessment methods, we found that all alien species occurring in roadsides showed high invasive potential and that these were the most abundant too. The SI was greater in more disturbed sites and no relationship was found between native and alien species diversity between sites with different
\end{abstract}


disturbance intensity associated with roadsides. We propose to take into consideration the most dangerous species in the park for mitigation plans. In addition to the WRA is a method that could be applied to conduct risk assessments in protected areas. Finally we highlight the role of roads in protected areas can be a major source of the spread of introduced species, increasing the severity of invasion.

KEYWORDS: Temperate rainforest, diversity and abundance, invasion ecology, alien plants, WRA.

\section{INTRODUCCIÓN}

La introducción de especies es una de las principales causas de pérdida de biodiversidad (Vitousek et al. 1997, Sala et al. 2000), por ende puede producir pérdida de recursos naturales y económicos a nivel mundial (Cox 1999, Chapin et al. 2000, Mack et al. 2000, Pimentel et al. 2005, Mooney et al. 2005). Este fenómeno puede ser promovido de forma intencional o accidental por la actividad antrópica (Drake et al.1989, Mooney \& Hobbs 2000, Richardson et al. 2000, Becerra et al. 2006), ya que las perturbaciones relacionadas con actividades humanas aumentan la presión de propágulos de especies introducidas (Lin et al. 2007, Pyšek et al. 2010). Además, la remoción de biomasa libera recursos y proporciona oportunidades para el establecimiento de estas especies (Higgins \& Richardson 1998, Pauchard $\&$ Alaback 2004). Por lo tanto, la intensidad y duración de estas perturbaciones pueden influir en el éxito de una especie introducida (Cole \& Landres 1996, Sher \& Hyatt 1999, Davis et al. 2000, Pauchard \& Alaback 2004, 2006). Esto también ocurre en lugares que tienen como objetivo la protección de la biodiversidad, como es el caso de las áreas protegidas, ya que en estos sitios las actividades turísticas, además de la formación de senderos y caminos, son una fuente importante de transporte y propagación de especies introducidas (McDonald et al. 1998, Pauchard \& Alaback 2004, Yates et al. 2004, Jiménez et al. 2013, Pauchard et al. 2013). Como consecuencia las áreas protegidas también han resultado ser vulnerables a la introducción de especies (Pauchard \& Alaback 2004, Domínguez et al. 2006, Yates et al. 2004, Jiménez et al. 2013, Pauchard et al. 2013).

Debido a las pérdidas biológicas y económicas que ocasiona la introducción de especies en una determinada región, diversos países y organizaciones gubernamentales se han preocupado de la prevención, manejo y erradicación de estas especies (Loockwood et al. 2007). Una de las metodologías utilizadas se denomina métodos de evaluación de riesgo, los cuales permiten evaluar y prevenir la introducción de especies que puedan establecerse exitosamente en una región geográfica (Pheloung et al.1999, Daehler et al. 2004, Kato et al. 2006, Křivánek \& Pyšek 2006, Daehler \& Denslow 2007, Gordon et al. 2008, Fuentes et al. 2010). En Australia se ha desarrollado un método de evaluación de riesgo llamado Australian Weed Risk Assessment, AWRA (en adelante WRA para referirnos al método, independiente del país o lugar donde se implemente), que tiene como objetivo evaluar el "potencial invasor" de una especie de planta (i.e. cuan riesgosa es la introducción de una especie en una región antes de que ésta sea introducida; o la probabilidad de volverse invasora) (Pheloung et al. 1999, Daehler et al. 2004, Kato et al. 2006, Křivánek \& Pyšek 2006, Gordon et al. 2008, Fuentes et al. 2010). Este método incorpora aspectos de tipo biogeográfico, así como biológicos y ecológicos de las especies en evaluación (Pheloung et al. 1999, Fuentes \& Pauchard 2011), por lo tanto considera la mayoría de los aspectos que estarían asociados al establecimiento exitoso de las especies introducidas en una determinada región geográfica (Baker 1974, Loockwood et al. 2007). Sin embargo, otros autores han utilizado este método cuando las especies ya se han introducido en un sitio, para implementar medidas de manejo (control y erradicación). En este caso se considera los atributos (valores WRA) que la hacen ser más riesgosa para el lugar, y la abundancia como medida de éxito de invasión en una región determinada (Daehler 2005). Además, este método permite generar índices para estimar y comparar cuán invadido se encuentra un sitio o comunidad determinada respecto a otra, lo que Daehler (2005) define como severidad de invasión (SI). Para determinar la severidad de invasión se utiliza la sumatoria del WRA por la abundancia de todas las especies introducidas en el sitio. Así, al tener dos sitios con diferentes niveles de severidad de invasión y dependiendo del valor conservativo de éste, los valores de SI proporcionan información que permitirían mejorar los planes de manejos para conservación de un área protegida (Daehler 2005).

Se ha postulado que comunidades con mayor riqueza de especies o con mayor diversidad de grupos funcionales tendrían menor probabilidad de ser invadidas (Elton 1958, Levine \& D'Antonio 1999, Lavorel et al. 1999, Shea \& Chesson 2002, Meiners \& Cadenasso 2005). Sin embargo, Shea \& Chesson (2002) proponen que esto es dependiente de la escala, pues a escala local existen investigaciones que describen que en algunas comunidades vegetales existe una relación negativa entre la riqueza de especies nativas e introducidas (Tilman 1997, Fargione et al. 2003). Se ha propuesto que esto ocurre porque la comunidad nativa estaría resistiendo el establecimiento de especies introducidas al utilizar todos los recursos; por lo que una especie introducida tendrá menor probabilidad de encontrar un nicho disponible (Tilman 1997, Shea \& Chesson 2002). Por lo tanto, en un espacio más reducido la competencia 
interespecífica sería inevitable, teniendo como efecto la exclusión competitiva de las especies introducidas (Shea \& Chesson 2002, Meiners \& Cadenasso 2005, Castro et al. 2007). Además, se ha descrito que las comunidades nativas que están en un estado sucesional tardío o en estado maduro tendrán mayor resistencia al proceso de invasión, pues serían comunidades que se encuentren en equilibrio, limitando el establecimiento de especies introducidas (Shea \& Chesson 2002). Por el contrario, Shea \& Chesson (2002) proponen que a escala regional puede existir una relación positiva entre la riqueza de plantas nativas e introducidas (Lonsdale 1999, Levine 2000, Sax 2002, Stohlgren et al. 1999, Meiners \& Cadenasso 2005, Castro et al. 2007), pues a macro escala existe mayor heterogeneidad ambiental y condiciones posibles para el desarrollo de especies nativas e introducidas. Este patrón refleja que los recursos y el espacio serían suficientes para la subsistencia de ambos tipos de especies (i.e. si el ambiente es capaz de sostener un gran número de especies nativas, entonces también puede sostener la presencia de especies introducidas).

En Chile existen ecosistemas con un alto valor de conservación debido a su riqueza y alto grado de endemismo, como es el caso de los bosques templados (Armesto et al. 1992, 1998). Esta condición ha despertado gran interés en su conservación por ser un hotspot de biodiversidad (Armesto et al. 1994). Dentro de este bioma, el Bosque Templado Lluvioso del Parque Nacional Puyehue forma parte de la Reserva de la Biosfera Bosques Templados Lluviosos de los Andes Australes (CONAF 2009). En este parque se han realizado estudios descriptivos que han mencionado la presencia de un total de 55 especies introducidas (MuñozSchick 1980, Mardones et al. 2012). Además, por esta área protegida atraviesa una carretera internacional con frontera en Argentina (Paso fronterizo Cardenal Antonio Samoré) con $53 \mathrm{~km}$ de camino (Muñoz-Schick 1980) y recibe una gran cantidad de visitantes (INE 2010). Esto podría estar aumentando el riesgo de introducción de especies (Pauchard et al. 2013). En consecuencia, dado el alto número de especies introducidas en esta área protegida de Bosque Templado Lluvioso, es importante conocer aspectos ecológicos como la SI y la relación entre la riqueza o diversidad de especies nativas e introducidas, para entender el proceso de invasión de un ecosistema nativo y puede también ser de utilidad para proponer planes de manejo efectivos y eficientes en este tipo de áreas de conservación. Además, debido a que existe una escasez de estudios que evalúen el riesgo que presentan las especies introducidas en los ambientes naturales en Chile, y la necesidad de determinar cuán invadida se encuentra una comunidad de bosque templado lluvioso, consideramos relevante evaluar en el piso vegetacional "Bosque laurifolio templado interior de Nothofagus dombeyi y Eucryphia cordifolia" del Parque Nacional Puyehue (Luebert \& Pliscoff 2006): i) ¿Qué especies introducidas presentan mayor potencial invasor?, ii) ¿Qué especies introducidas son las que presentan mayor éxito de invasión?, iii) ¿Las especies con mayor potencial invasor, son a su vez las más exitosas?, iv) ¿Sitios sometidos a alta frecuencia e intensidad de perturbación presentarán mayor severidad de invasión?, v) ¿Existe una relación negativa entre la diversidad de especies nativas e introducidas (a nivel local) en sitios con diferente nivel de perturbación?

\section{MATERIALES Y MÉTODOS}

AREA DE ESTUdio y MUESTREO DE COMPOSICIÓN Y ABUNDANCIA El Parque Nacional Puyehue se ubica en la comuna de Puyehue, Chile, entre los $40^{\circ} 26^{\prime}$ y $40^{\circ} 58^{\prime} \mathrm{S}$ y $72^{\circ} 22^{\prime} \mathrm{y}$ $71^{\circ} 50^{\prime} \mathrm{O}$ (CONAF, 2009). Tiene un promedio anual de precipitaciones de $450 \mathrm{~mm}$ y una temperatura media anual de 9,2 ${ }^{\circ} \mathrm{C}$ (Muñoz-Schick 1980). El muestreo se desarrolló en el sector de Anticura, correspondiente al piso vegetacional, denominado "Bosque laurifolio templado interior de Nothofagus dombeyi y Eucryphia cordifolia" (Luebert \& Pliscoff 2006).

Para la determinación de la composición y abundancia de especies introducidas y nativas, se realizaron 34 parcelas (10 m x $2 \mathrm{~m}$ ), divididas en dos transectos de 1×10 metros, subdivididos a su vez en 10 cuadrantes de $1 \mathrm{x} 1$ metro. El muestreo consideró la distinción de dos ambientes en relación a su grado de uso o perturbación actual (17 parcelas por cada uno): sitio tipo "A" con alto nivel de perturbación (camino para turistas, cercano a zona de camping y veranada) y sitio tipo "B" con bajo nivel de perturbación (antiguo camino, cerrado actualmente para turistas desde la década del 60). Es importante mencionar que el uso del sitio B antes de ser clausurado fue relativamente intenso, pues era el camino principal que conectaba con la frontera Argentina, por ende era utilizado con mucho mayor frecuencia que hoy en día.

Producto del muestreo se colectaron 72 especímenes de plantas (entre nativas e introducidas), las cuales fueron identificadas y depositadas en la colección del Herbario de la Universidad de Concepción (CONC). La abundancia fue estimada como porcentaje de cobertura de cada especie en cada cuadrante, utilizando una escala de 6 categorías modificada a partir del método de Braun-Blanquet (i.e. $0 \%$ : especie ausente en el cuadrante; 5\%: presencia entre $1-5 \%$; 25\%: entre 6-25\%; 50\%: entre un 26-50\%; 75\%: entre un $51-75 \%$ y 100\%: entre un 76-100\%). La diversidad $(\mathrm{H})$ se calculó con el índice de Shannon Weiner.

DETERMINACIÓN DEL VALOR DE POTENCIAL INVASOR Y SEVERIDAD DE INVASIÓN

La determinación del WRA de cada especie introducida se realizó en base al trabajo de Pheloung et al. (1999). El cual consiste en responder un cuestionario de 49 preguntas, considerando aspectos relacionados con la ecología, biología reproductiva y características morfológicas de cada 
especie introducida. Cada respuesta tiene un determinado valor numérico, el puntaje de WRA será la suma de dichos valores. Si el resultado de la sumatoria es cero o menos, la especie tiene poca probabilidad de ser invasora. Si la puntuación es mayor a 6 , tiene un alto "potencial invasor" y es riesgosa para el sitio en estudio. Si la puntuación está entre 1 y 6 , se requiere mayor información antes de tomar una decisión, esto quiere decir, que se debe tratar de abarcar una mayor cantidad de preguntas del cuestionario y realizar una segunda evaluación (Pheloung et al. 1999). Para aplicar este sistema de evaluación a las especies presentes en este estudio, se siguió la recomendación propuesta por Gordon et al. (2010), quienes detallan cada una de las 49 preguntas del cuestionario, para su mejor comprensión y uso. Además, debido a que el área de estudio corresponde al bosque templado siempreverde de Chile (por lo que tiene ciertas condiciones climáticas características), se realizaron cambios, basados en el trabajo de Fuentes et al. 2010 (WRA-Ch), por lo tanto se modificaron las preguntas biogeográficas o las que tienen relación con el lugar de estudio, específicamente se modificaron las preguntas: a) Pregunta 2.01: ¿Las especies se han adaptado en lugares con clima Australiano?, por: ¿La especie se ha adaptado en lugares con clima de bosque templado?, b) Pregunta 2.04: ¿La especie es nativa o naturalizada en lugares con periodos largos de sequía?, por: ¿La especie es nativa o naturalizada en lugares con estaciones bien definidas?, c) Pregunta 4.10: ¿La especie crece en suelos infértiles?, por: ¿La especie crece en ambientes perturbados?, d) Pregunta 8.05: ¿La especie tiene enemigos naturales en Australia?, por: ¿La especie tiene enemigos naturales en el sur de Chile?

Para el cálculo de la severidad de invasión en los sitios de muestreo (i.e. sitios A, B y total), se calculó el índice de severidad de invasión por parcela, lo cual indica cuán invadida se encuentra un área (Daehler 2005).

$$
\text { Severidad de invasión }=\sum_{i=1}^{n} S_{i} A_{i}
$$

Donde $\mathrm{Si}$ corresponde al puntaje obtenido con el sistema WRA para cada especie de planta introducida y $A_{i}$ es su abundancia (porcentaje de cobertura) en un área determinada. El resultado es la suma de ambos productos. En general, la severidad de la invasión es alta cuando muchas especies introducidas están presentes, si se encuentran en gran cantidad (abundancia es alta), o si las especies presentes tienen puntuaciones altas de WRA (Daehler 2005).

\section{ANÁlisis DE DATOS}

Para evaluar si existe una relación lineal positiva entre la abundancia (porcentaje de cobertura) y los valores de WRA de las especies introducidas, se realizó una correlación lineal de Pearson. Además se realizó una comparación entre los valores de abundancia, WRA y SI de los sitios tipo A y B y se realizó una prueba t de Student para corroborar si las diferencias encontradas eran estadísticamente significativas. Por otra parte, con el fin de evaluar cuales de las especies más abundantes son la que marcan la diferencia en las SI, se realizó una comparación estadística (prueba t de Student) de las cuatro especies que presentaron mayores porcentajes de cobertura entre sitios (A y B).

Finalmente, se evaluó el tipo de relación (lineal, logística y exponencial) entre la diversidad de especies introducidas y nativas en los sitios con diferentes niveles de perturbación (sitio tipo A y B). Los datos se ajustaron a una relación lineal, por lo que se realizó una correlación lineal de Pearson entre la diversidad de especies introducidas y nativas para cada uno de los sitios. Se observó la magnitud (valor de la pendiente) y se compararon los valores de $r$. Estos análisis fueron realizados en el programa Statistica 10.

\section{RESULTADOS}

Se encontró un total de 24 especies introducidas en los sitios de muestreo, correspondiente al 43,6\% del total de especies introducidas del parque. Los resultados muestran que, mediante los valores obtenidos por el método de evaluación de riesgo (Weed Risk Assessment), todas las especies presentaron valores de WRA mayores a 6 . El promedio de los valores de WRA fue 29. Las especies que presentaron mayores valores de WRA (sobre el promedio) fueron: Conium maculatum L., Ranunculus repens L., Rubus constrictus P.J. Müll. \& Lefèvre, Rosa rubiginosa L., Rumex obtusifolius L., Prunella vulgaris L., Rumex acetosella L., Holcus lanatus L., Lotus pedunculatus Cav., Anthoxanthum odoratum L., Trifolium repens L., Taraxacum officinale F. H. Wigg., Arrhenatherum elatius (L.) P. Beauv. ex J. Presl \& C. Presl, Hypochaeris radicata L. y Plantago lanceolata L. (Anexo 1). En cuanto a la abundancia, la especie que presentó mayor valor para ambos sitios de muestreo fue Ranunculus repens (Fig. 2). Por otra parte, cabe destacar que las especies Holcus lanatus y Anthoxanthum odoratum son muy abundantes sólo en el sitio A, mientras que Prunella vulgaris es la más abundante en el sitio B (Fig. 2). Al evaluar la correlación entre WRA y abundancia de las especies introducidas (Fig. 1), encontramos una relación lineal positiva estadísticamente significativa $(\mathrm{r}=0,42 ; p=0,04)$.

La comparación del índice de severidad de invasión entre los A y B muestra que la SI en el sitio con menor intensidad de perturbación (sitio B), fue significativamente menor que la severidad de invasión del sitio con alta intensidad de perturbación o sitio A ( $\mathrm{t}=2,92 ; p=0,006$; ver Fig. $3 \mathrm{a})$. La abundancia (cobertura) fue significativamente mayor en las especies introducidas en el sitio A (Fig. 3b), sin embargo no existen diferencias significativas entre ambos sitios en el 


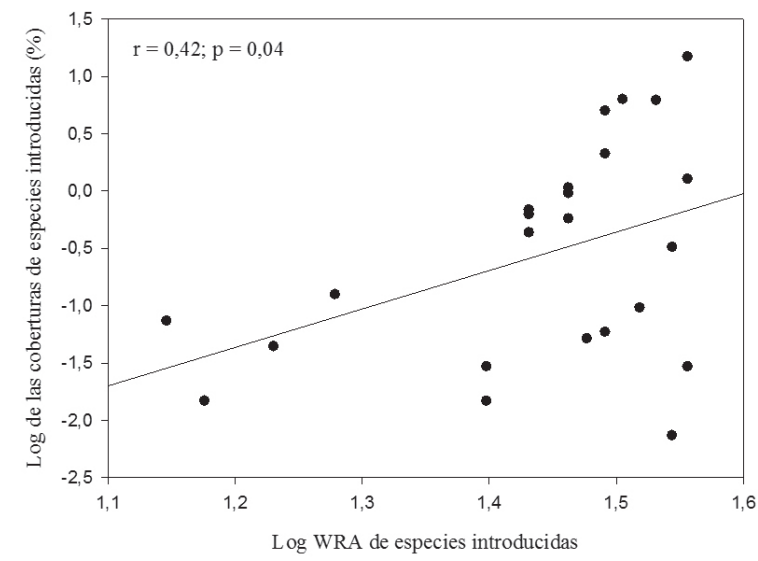

FIgURA 1. Gráfico de dispersión que representa la correlación lineal entre los valores de Log de WRA y Log de los porcentajes de cobertura de las especies introducidas en bordes de caminos del Parque Nacional Puyehue.

FIGURE 1. Scatter plot showing the linear correlation between the values of Log WRA and Log the percentage coverage of introduced species in roadsides of Puyehue National Park.

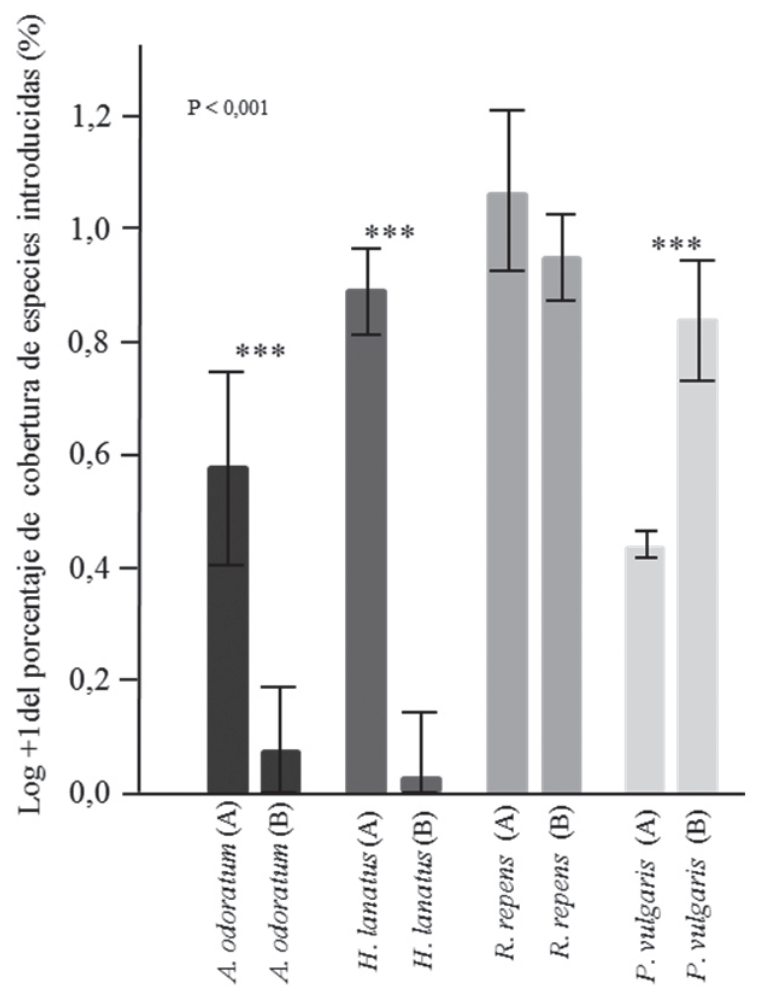

FIGURA 2. Valores promedio ( $\pm \mathrm{ES}$ ) de los porcentajes de cobertura $(\%)$ de las especies introducidas más abundantes entre sitios con diferentes niveles de perturbación (A y B).

FIGURE 2. Mean values ( \pm SE) of the coverage percentages $(\%)$ of introduced species more abundant among sites with different levels of disturbance (A and B).

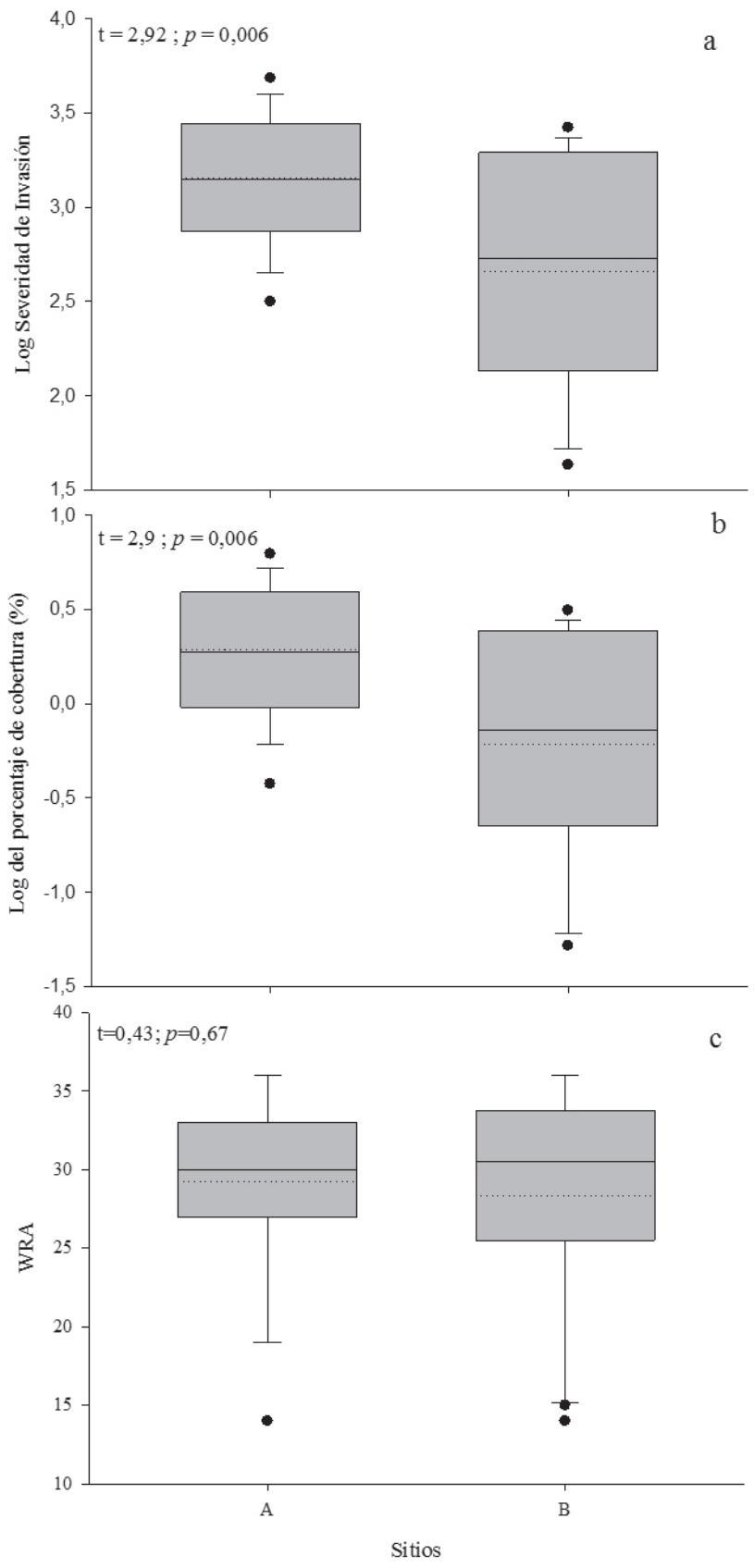

Figura 3. Comparación de promedios de los sitios A y B de las diferentes variables consideradas en este estudio. Media (línea punteada), mediana (línea entera), percentiles $10^{\circ}, 25^{\circ}, 75^{\circ} \mathrm{y}$ $90^{\circ}$ de a) severidad de invasión, b) porcentaje de cobertura, y c) Valores de WRA de las especies introducidas en sitios con diferentes niveles de perturbación (A, alto; B, bajo).

Figure 3. Comparison of average of the sites A and B of different variables considered in this study. Mean (dotted line), median (solid line), $10^{\text {th }}, 25^{\text {th }}, 75^{\text {th }}$ and $90^{\text {th }}$ percentiles of a) severity of invasion, b) percentage coverage of introduced species, and c) WRA values of introduced species in sites with different levels of disturbance (A, high; and B, low). 
valor promedio de WRA de las especies (Fig. 3c). Además, al hacer la estimación de la relación entre la diversidad de especies nativas e introducidas por sitios, ninguno de los dos sitios muestra relación lineal entre dichas variables (Sitio A: $\mathrm{r}=0,02, p=0,62$ y Sitio B: $\mathrm{r}=0,003, p=0,81$ respectivamente).

\section{DISCUSIÓN}

Todas las especies introducidas muestreadas en el parque tuvieron valores de WRA mayor a 6, lo que desde la perspectiva del método de evaluación de riesgo significa que todas estas especies son potencialmente invasoras (Pheloung et al. 1999), para el piso vegetacional "Bosque laurifolio templado interior de Nothofagus dombeyi y Eucryphia cordifolia" del Parque Nacional Puyehue. Estos resultados son esperables ya que Mardones et al. (2012) reportaron estas especies como naturalizadas en el parque. Esto quiere decir que son especies introducidas establecidas que posiblemente han logrado reproducirse y/o dispersarse en este nuevo hábitat (sensu Richardson et al. 2000). Por lo tanto, las especies introducidas con mayores valores de WRA corresponden a las especies con mayor potencial invasor para el parque, pudiendo representar una validación del método de evaluación de riesgo aplicado a una zona específica del ecosistema bosque templado, que además se encuentra dentro de un área protegida. Según Fuentes et al. (2014), la mayoría de estas especies, también son consideradas invasoras en Chile: Rosa rubiginosa (WRA = 24), Ranunculus repens (WRA = 23), Conium maculatum (WRA = 22), Rumex acetosella (WRA = 21), Holcus lanatus $(\mathrm{WRA}=19)$, Trifolium repens $(\mathrm{WRA}=19)$, Hypochaeris radicata $(\mathrm{WRA}=15)$, Plantago lanceolata $(\mathrm{WRA}=$ 15), Anthoxanthum odoratum (WRA = 13), Taraxacum officinale (WRA =13), y Prunella vulgaris (WRA =12). En nuestro trabajo, la especie introducida que presentó mayor valor de WRA y además mayor abundancia para ambos sitios es Ranunculus repens. Por lo tanto, esta es la especie con mayor éxito invasor en bordes de camino del piso vegetacional estudiado. Este éxito invasor puede deberse a que tiene las características biológicas y ecológicas que les otorgarían mayor éxito de invasión (Pheloung et al. 1999). Por ejemplo, Ranunculus repens presenta reproducción vegetativa y a través de semillas, dispersión por viento, agua y animales, semillas viables por largos periodos de tiempo y colonización y ocupación rápida de zonas abiertas (Fuentes et al. 2014). Según sus características biogeográficas, esta especie también es considerada invasora en otros lugares de bosque templado y de Chile (Fuentes et al. 2014). Además, de acuerdo a la clasificación de Matthei (1995), Ranunculus repens está clasificada como "Maleza principal" (i.e. aquella que significa daños importantes en agricultura, pero nunca la pérdida total de un cultivo). Si bien, las categorías de Matthei (1995) están basadas en el efecto de las especies introducidas en cultivos, Fuentes (datos no publicados) encontró que estas categorías se relacionan positivamente con los valores de WRA en Chile, por lo que se sugiere tener especial atención con esta especie para realizar planes de manejo en el parque. Sin embargo, aún es necesaria la realización de otros estudios que evalúen los impactos de Ranunculus repens al introducirse en un hábitat nativo.

Hay especies que fueron muy abundantes y están presentes solo en el sitio con alto nivel de perturbación (sitio A), como: Anthoxanthum odoratum y Holcus lanatus, mientras que otras como Prunella vulgaris fue más abundante en el sitio con bajo nivel de perturbación (sitio B). Esto podría deberse a que existen diferencias en la presión de propágulos de estas especies en los diferentes sitios (Colautti et al. 2006). También las diferencias en abundancia (porcentaje de cobertura) de estas especies (entre sitios) puede deberse a las características de cada especie en cuanto a cómo responden al uso histórico de ambos sitios. Por ejemplo, se sabe de literatura que la germinación de Anthoxanthum odoratum es estimulada por las perturbaciones (Fuentes et al. 2014) y Holcus lanatus es dispersada por actividades humanas (Fuentes et al. 2013), lo que puede beneficiar la mayor cobertura en el sitio con alta perturbación (sitio A). Mientras que Prunella vulgaris puede establecerse en hábitats nativos (Godoy et al. 2010), lo que podría explicar su abundancia en hábitats menos perturbados.

En este estudio encontramos especies con alto WRA, que aún no son tan abundantes (menor porcentaje de cobertura) como: Conium maculatum, Rosa rubiginosa, Rubus constrictus y Rumex obtusifolius. Esto quiere decir que son especies con alto potencial invasor que aún no son exitosas, pero con alta probabilidad de serlo (Daehler 2005). Esto se puede deber a que las tres primeras llevan menos tiempo en el parque, pues corresponden a registros recientes (Mardones et al. 2012). En este sentido, el tiempo de residencia de una especie en un lugar se ha propuesto como un factor importante en la determinación del éxito de invasión de una especie introducida (Rozenfields \& Mackenzie 1999, Rejmánek 2000, Pyšek et al. 2003, Castro et al. 2005, Wilson et al. 2007). Es por ello, que se sugiere mantener un seguimiento de estas plantas, pues en un futuro podrían alcanzar el equilibrio y ocupar los lugares que potencialmente son habitables por estas especies (sensu Peña-Gómez et al. 2014). Predecir qué especies tienen un alto potencial de invasión ha sido objetivo de variados estudios y debates de la comunidad científica (Hulme 2003). El hecho de encontrar una relación positiva entre las especies con mayor potencial invasor y las especies con mayor éxito de invasión (medido como cobertura) en nuestro sitio de estudio (Parque Nacional Puyehue), podrían indicar que el índice WRA, podría ser buen indicador del potencial invasor de las especies introducidas (Pheloung et al. 1999, Daehler et al. 2004, Kato et al. 2006, Krivánek \& 
Pyšek 2006, Daehler \& Denslow 2007, Gordon et al.2008), inclusive en Chile (Fuentes et al. 2010).

La mayor severidad de invasión que presenta el sitio más perturbado (sitio A) se debe a que el sitio A presenta valores significativamente mayores de abundancia de especies de plantas introducidas que el sitio B (Fig. 2b), pues la SI es dependiente de las abundancias y los valores de WRA (Daehler 2005). Esta diferencia en SI podría deberse particularmente a las mayores abundancias de las especies Anthoxanthum odoratum y Holcus lanatus en el sitio con alto nivel de perturbación o sitio A (Fig. 2). A su vez, la SI podría estar asociada a que el sitio A presenta una mayor frecuencia de perturbación antrópica, debido al alto tránsito de turistas y ganado en esta zona del estudio. Este resultado podría relacionarse con los supuestos propuestos para la ecología de invasiones, donde las perturbaciones de tipo antrópica son consideradas una de las principales fuentes de propagación de las especies introducidas (Cole \& Landres 1996, Sher \& Hyatt 1999, Davis et al. 2000, Shea \& Chesson 2002, Pauchard $\&$ Alaback 2004, 2006), y donde la frecuencia e intensidad de este fenómeno estaría estrechamente relacionado con el grado y duración de la intervención humana (Vidal 2005). En este sentido, los caminos han sido propuestos como un elemento importante para la dispersión de propágulos y el establecimiento de especies introducidas (Pauchard \& Alaback 2004, Lin et al. 2007, Pyšek et al. 2010, Jiménez et al. 2013, Pauchard et al. 2013), lo que puede traer consecuencias negativas para las áreas a conservar (Trombulak \& Frissell 2000). Por otra parte, la menor severidad de invasión que presenta el sitio menos perturbado (sitio B), probablemente se deba a que este sitio ya se encuentra clausurado (no se permite su uso en el parque), por lo que la frecuencia de perturbación antrópica debido a la visita de turistas es menor. Este hecho podría haber disminuido la dispersión y la llegada de nuevos propágulos de plantas introducidas, conllevando a que este sitio se encuentre con menor SI y abundancia de especies introducidas, lo que podría permitir una recuperación paulatina de la comunidad nativa (sensu Yates et al. 2004).

Respecto a la relación de la diversidad (a escala local) entre especies nativas e introducidas, el no encontrar una relación entre estos grupos (considerando diferentes niveles de perturbación), podría significar que la presencia de especies nativas e introducidas en el área perturbada estaría dada por una dispersión y establecimiento más bien azaroso. Por lo tanto, actualmente el ambiente estaría sustentando a una comunidad que combina especies nativas e introducidas, sin evidencias claras de interacciones entre ellas que determinen el desmedro en la diversidad de un grupo en función del otro, probablemente debido a una amplia disponibilidad de recursos en los sitios de estudio (Meiners \& Cadenasso 2005, Castro et al. 2007). Esto podría explicarse debido a que ambos sitios corresponden a bordes de caminos, por lo tanto, a pesar de trabajar a escala local (donde probablemente los recursos serían más limitantes y la interacción debería ser negativa), se les está otorgando el espacio disponible para no competir por recursos (Shea \& Chesson 2002). De esta manera, tanto las plantas introducidas como las nativas estarían utilizando los nichos disponibles dejados por la fragmentación del hábitat, ya que los caminos son considerados hábitats propicios particularmente para el establecimiento de especies de plantas introducidas (Trombulak \& Frissell 2000, Arévalo et al. 2005, Seipel et al. 2012, Jiménez et al. 2013), pero para este caso, también para especies nativas dada la matriz vegetacional en la que se encuentran estos caminos.

Finalmente las abundancias (porcentaje de cobertura) y los valores de WRA nos permitirían establecer qué especies de plantas introducidas pueden ser más riesgosas y exitosas en un determinado lugar, y además permitiría comparar la severidad de invasión entre sitios con diferente grado de perturbación en áreas protegidas de bosque templado como en el caso del Parque Nacional Puyehue. Este tipo de estudios ayuda a determinar cuáles especies debieran ser prioridad para su erradicación en planes de manejo (Daehler 2005). Aunque Gardener et al. (2011) sugieren que producto de la globalización la introducción de especies es inevitable, es importante la prevención efectiva y la realización de inventarios de estas especies, para que estos procesos sean más lentos o retenidos (Fuentes et al. 2013). Sin embargo, aún faltan estudios que ayuden a determinar un buen manejo de las áreas protegidas en este sentido. Nuestros resultados sugieren que los ambientes perturbados del Parque Nacional Puyehue son susceptibles al ingreso de especies con altos potencial invasor (i.e. WRA), debido a la vía de colonización que ofrecen los caminos. Por otra parte, si bien nuestros resultados no muestran que en los sitios muestreados el componente nativo oponga resistencia al establecimiento de especies de plantas introducidas, estudios anteriores en este ecosistema forestal muestran que existen especies que han sido capaces de colonizar y establecerse en el sotobosque del Parque Nacional Puyehue (e.g. Prunella vulgaris; Godoy et al. 2010). Por lo tanto, es necesario entender mejor los procesos ecológicos y los impactos de las invasiones biológicas en estos ecosistemas. Trabajos como éste ayudan a entender los patrones de diversidad y abundancia que tendrían las especies introducidas en un área protegida en el bosque templado lluvioso.

\section{AGRADECIMIENTOS}

Quisiéramos agradecer especialmente a la Dra. Maritza Mihoc por la gran ayuda brindada en este trabajo. Además agradecer a Álvaro Zúñiga por las sugerencias y revisiones al manuscrito y a Rafaela Mardones por la ayuda prestada. DM agradece a Beca CONICYT 22131484. Este estudio fue financiado por el proyecto FONDECYT $1140455 \mathrm{y}$ FONDECYT 3120125 , 


\section{BIBLIOGRAFÍA}

Arévalo, J.R., J.D. Delgado, R. Otto, A. Naranjo, M. Salas \& J.M. Fernández-Palacios. 2005. Distribution of alien vs. native plant species in roadside communities along an altitudinal gradient in Tenerife and Gran Canaria (Canary Islands). Perspectives in Plant Ecology Evolution and Systematics 7: 185-202.

Armesto, J.J., C. Smith-Ramírez, P. León \& M.T.K. Arroyo. 1992. Biodiversidad y conservación del bosque templado en Chile. Ambiente y Desarrollo 8: 19-24.

Armesto, J.J., C. Villagrán \& C. Donoso. 1994. Desde la era glacial a la industrial: La historia del bosque templado chileno. Ambiente y Desarrollo 10(1): 66-72.

Armesto, J.J., R. Rozzi, C. Smith-Ramírez \& M.T.K. Arroyo. 1998. Conservation targets in South American temperate forests. Science 282: 1271-1272.

BAKer, H. 1974. The evolution of weeds. Annual Reviews of Ecology and Systematics 5: 1-24.

BECERRA, P.I. 2006. Invasión de árboles alóctonos en una cuenca preandina de Chile central. Gayana Botánica 63(2): 161-174.

Castro, S.A., J.A. Figueroa, M. Muñoz-Schick \& F.M. Jaksic. 2005. Minimum residence time, biogeographic origin, and life cycle as determinants of the geographical extent of naturalized plants in continental Chile. Diversity and Distributions 11: 183-191.

Castro, S.A., L. Marone \& F.M. Jaksic.2007. Invasiones. En: F.M. Jaksic \& L. Marone (eds.). Ecología de comunidades, pp. 217-229. Ediciones Universidad Católica de Chile, Santiago, Chile.

Chapin, F.S., E.S. Zavaleta, V.T. Eviner, R.L. Naylor, P.M. Vitousek, H.L. Reynolds, D.U. Hooper, S. Lavorel, O.E. Salai, S.E. Hobbie, M.C. Mack \& S. Díaz. 2000. Consequences of changing biodiversity. Nature 405: 234242.

Colautti, R.I., I.A. Grigorovich \& H.J. MacIsaac. 2006. Propagule pressure: a null model for biological invasions. Biological Invasions 8: 1023-1037.

Cole, D. \& P. Landres. 1996. Threats to wilderness ecosystems: impacts and research needs. Ecological Applications 6(1): 168-184.

CONAF. 2009. Parque Nacional Puyehue. Cuenta pública. 2009. Ministerio de Agricultura. Santiago, Chile.14 pp.

Cox, G.W. 1999. Alien species in North America and Hawaii. Island Press, Washington D.C. 388 pp.

DAeHLER, C. 2005. Invasive species. In: D. Mueller-Dombois, K.W. Bridges \& C. Daehler (eds.), Biodiversity assessment of tropical island ecosystems, pp. 197-208. PABITRA manual for interactive ecology and management. Honolulu, Hawaii.

Daehler, C.C. \& J.S. Denslow. 2007. The Australian weed risk assessment system: does it work in Hawaii? Would it work in Canada? In: D.R. Clements \& S.J. Darbyshire (eds.), Invasive plants: inventories, strategies and action-Topics in Canadian Weed Science. pp. 9-24. Canadian Weed Science Society, Québec.

Daehler, C., J.S. Denslow, S. Ansari \& H. Kuo. 2004. A risk assessment system for screening out harmful invasive pest plants from Hawaii and other Pacific Islands. Conservation Biology 18: 360-368.

Davis, M.A., J.P. Grime \& K. Thompson. 2000. Fluctuating resources in plant communities: a general theory of invasibility. Journal Ecology 88: 528-534.

Domínguez, E., A. Elvebakk, C. Marticorena \& A. Pauchard. 2006. Plantas introducidas en el Parque Nacional Torres del Paine, Chile. Gayana Botánica 63(2): 131-141.

Drake, J, H.A. Mooney, F. Di Castri, R. Groves, F. Kruger, M. ReJMANEK \& M. WiLliamson. 1989. Biological Invasions: a global perspective. Chichester (UK): John Wiley \& Sons. $525 \mathrm{pp}$.

ELton, C.S. 1958. The ecology of invasions by animals and plants. Methuen, London. $181 \mathrm{pp}$.

Fargione, J., C.S. Brown \& D. Tilman. 2003. Community assembly and invasion: An experimental. Proceedings of the National Academy of Science USA 100(15): 8916-8920.

Fuentes, N. \&A. PAuchard. 2011. Evaluación de Riesgo de especies de Plantas Introducidas (ERPI) en Chile: estableciendo prioridades para su manejo o control. Documento técnico 1. Laboratorio de Invasiones Biológicas (LIB), Concepción, Chile. 21 pp.

Fuentes, N., A. Pauchard, P. SÁnchez, J. Esquivel \& A. Marticorena. 2013. A new comprehensive database of alien plant species in Chile based on herbarium records. Biological Invasions 15(4): 847-858.

Fuentes, N., P. Sánchez, A. Pauchard, J. Urrutia, L. Cavieres \& A. Marticorena. 2014. Plantas invasoras del centro-sur de Chile: Una guía de campo. Laboratorio de Invasiones biológicas (LIB). Concepción, Chile. 276 pp.

Fuentes, N., E. Ugarte, I. Kuhn \& S. Klotz. 2010. Alien plants in southern South America. A framework for evaluation and management of mutual risk of invasion between Chile and Argentina. Biological Invasions 12: 3227-3236.

Gardener, M.R., R. O. Bustamante, I. Herrera, G. Durigan, V.R. Pivello, M.F. Moro, A. Stoll, B. Langdon, Z. Baruch, A. Rico, A. Arredondo-Núñez \& S. Flores. 2011. Plant invasions research in Latin America: fast track to a more focused agenda. Plant Ecology \& Diversity 5(2): 225-232.

Godoy, O., A. Saldaña, N. Fuentes, F. Valladares \& E. Gianoli. 2010. Forests are not immune to plant invasions: phenotypic plasticity and local adaptation allow Prunella vulgaris to colonize a temperate evergreen rainforest. Biological Invasions 13: 1615-1625.

Gordon, D., D. Onderdonk, A. Fox \& R.Stocker. 2008. Consistent accuracy of the Australian weed risk assessment system across varied geographies. Diversity and Distribution 14 : 234-242.

Gordon, D.R., B. Mitterdorfer, P.C. Pheloung, S. Ansari, C. Buddenhagen, C. Chimera, C. Daehler, W. Dawson, J. Denslow, T.N. Jaqualine, A. Larosa, T. Nishida, D.A. Onderdonk, D. Panetta, P. Pyšek, R. Randall, D.M. Richardson, J. Virtue \& P. Williams. 2010. Guidance for addressing the Australian Weed Risk Assessment questions. Plant Protection Quarterly 25(2): 56-74.

Higgins, S.I. \& D.M. Richardson. 1998. Pine invasions in the southern hemisphere: modelling interactions between organism, environment and disturbance. Plant Ecology 135: 79-93.

Hulme, P. 2003. Biological invasions: winning the science battles but losing the conservation war? Oryx 37:178-193.

INE ChILE. 2010. Turismo informa Anual 2010. SERNATUR Ministerio de Economía, Fomento y Turismo. Chile. 119 pp.

Jiménez, A., A. Pauchard, A. Marticorena \& R.O. Bustamante. 2013. Patrones de distribución de plantas introducidas 
en áreas silvestres protegidas y sus áreas adyacentes del centro-sur de Chile. Gayana Botánica 70(1): 110-120.

Kato, H., K. НатA, Н. Yамамото \& T. Yoshioka. 2006. Effectiveness of the weed risk assessment system for the Bonin Islands. In: F. Koike, M.N. Clout, M. Kawamichi, M. de Poorter \& K. Iwatsuki (eds.), Assessment and Control of Biological Invasion Risks, pp. 65-72. Shoukadoh Book Sellers, Kyoto, Japan and IUCN, Gland, Switzerland.

KrIVÁneK, M. \& P. PYŠEK. 2006. Predicting invasions by woody species in a temperate zone: a test of three risk assessment schemes in the Czech Republic (Central Europe). Diversity and Distributions 12: 319-327.

Lavorel, S., A. Priur-Richard \& K. Grigulis. 1999. Invasibility and diversity of plant communities: from patterns to processes. Diversity Distribution 5: 41-49.

LEVINE, J.M. 2000. Species diversity and biological invasions: relating local process to community pattern. Science 288 : 852-854.

LeVINE, J.M. \& C.M. D’Antonio. 1999. Elton revisited: a review of evidence linking diversity and invasibility. Oikos 87 : $15-26$.

Lin, W., G. Zhou, X. Cheng \& R. Xu. 2007. Fast Economic Development Accelerates Biological Invasions in China. Plos One 2(11): e1208.

Lockwood, J., M. Hoopes \& M. Marchetti. 2007. Invasion Ecology. Blackwell Publishing Ltd. Massachusetts, USA. $304 \mathrm{pp}$.

Lonsdale, W.M. 1999. Global patterns of plant invasions and the concept of invisibility. Ecology 80(5): 1522-1536.

Luebert, F. \& P. Pliscoff. 2006. Sinopsis bioclimática y vegetacional de Chile. Editorial Universitaria. Santiago, Chile. 316 pp.

Mack, R.N., D. Simberloff, W.M Lonsdale, H. Evans, M. Clout \& F. Bazzaz. 2000. Biotic invasions: Causes, epidemiology, global consequences and control. Ecological Applications 10: 689-710.

Mardones, D., A. Zúñiga-Reinoso, M. Mihoc \& A. Saldaña. 2012. Flora introducida asociada a bordes de senderos en el bosque templado siempreverde: nuevos registros para el Parque Nacional Puyehue (Región de Los Lagos, Chile). Chloris Chilensis, año 15, N². URL: http:www. chlorischile.cl

Matthei, O. 1995. Manual de las malezas que crecen en Chile. Alfabeta Impresores, Santiago de Chile. 545 pp.

McDonald, I.A.W, D.M. Graber, S. DeBenedettil, R.H. Groves \& E.R. FuEnTES. 1998. Introduced species in nature reserves in Mediterranean-type climatic regions of the world. Biological Conservation 44: 37-66

Meiners, S. \& M. Cadenasso. 2005. The relationship between community diversity and exotic plants: cause or consequence of invasion? En: Inderjit (eds.), Invasive Plants: Ecological and Agricultural Aspects, pp. 97-127. Birkhäuser, Suiza.

Mooney, H.A. \& H.A. Hobbs. 2000. Invasive Species in a Changing World. Island Press, California. 472 pp.

Mooney, H.A., R.N. Mack, J.A. McNeely, L.E Neville, P.J. Schei \& J.K. WAAge. 2005. Invasive alien species: a new synthesis. Island Press, Washington DC. 368 pp.

Muñoz-Schick, M. 1980. Flora del Parque Nacional Puyehue. Editorial Universitaria, Santiago de Chile. 558pp.

Pauchard, A. \& P.B. Alaback. 2004. Influence of elevation, land use, and landscape context on patterns of alien plant invasions along roadsides in protected areas of southcentral Chile. Conservation Biology 18: 238-248.

Pauchard, A. \& P.B. Alaback. 2006. Edge type defines alien plant species invasions along Pinus contorta burned, highway and clearcut forest edges. Forest Ecology and Management 223: 327-335.

Pauchard A., N. Fuentes, A. Jiménez, R. Bustamante \& A. MarticorenA. 2013. Alien Plants Homogenise Protected Areas: Evidence from the Landscape and Regional Scales in South Central Chile. In: L.C. Foxcroft, P. Pyšek, D.M. Richardson \& P. Genovesi (eds.), Plant Invasions in Protected Areas: Patterns, Problems and Challenges, Invading Nature, pp. 191-208. Springer Series in Invasion Ecology 7 DOI 10.1007/978-94-007-7750-7_10

Peña-Gómez, F.T., P.C. Guerrero, G. Bizama, M. Duarte \& R.O. Bustamante. 2014. Climatic Niche Conservatism and Biogeographical Non-Equilibrium in Eschscholzia californica (Papaveraceae), an Invasive Plant in the Chilean Mediterranean Region. PlosOne 9(8): e105025. doi:10.1371/journal.pone.0105025.

Pheloung, P.C., P.A Williams \& S.R. Halloy. 1999. A weed risk assessment model for use as a biosecurity tool evaluating plant introductions. Journal of Environmental Management 57: 239-251.

Pimentel, D., R. ZúÑIga \& D. Morrison. 2005. Update on the environmental and economic costs associated with alieninvasive species in the United States. Ecological Economic 52: 273-288.

PyŠEK, P., S. Jiri, M. BohumIL \& V. JARosik. 2003. Czech alien flora and the historical pattern of its formation: what came first to Central Europe? Oecología 135: 122-130.

Pyšek, P., V. Jarošík, P.E. Hulme, I. Kühn, J. Wild, M. Arianoutsou, S. Bacher, F. Chiron, V. Didžiulis, F. Essl, P. Genovesis, F. Gherardik, M. HejdaA, S. Karkl, P.W. Lambdonm, M.L. Desprez-Loustaun, W. Nentwigo, J. Pergla, K. Poboljšajp, W. Rabitschi, A. Roquesq, D.B. Royr, S. Shirleys, W. Solarzt, M. Vilàu \& M. Winte. 2010. Disentangling the role of environmental and human pressures on biological invasions across Europe. Proceedings of the National Academy of Science USA 107: 12157-12162.

REJMÁNEK, M. 2000. Invasive plant: approaches and predictions. Austral Ecology 25: 497-506.

Richardson, D.M., P. Pyšek, M. Rejmánek, M.G. Barbour, F.D. PAnetTA \& C.J. West. 2000. Naturalization and invasion of alien plants: concepts and definitions. Diversity and Distributions. Ecological Society of America 6: 93-107.

Rozenfields, A.C.F. \& R. Mackenzie. 1999. The weed invasion in Tasmania in the 1870s: knowing the past to predict the future. In: A.C. Bishop, M. Boersma \& C. Barnes (eds.), 12th Australian weed conference papers and proceedings, pp. 581-583. CSIRO Publications, Hobart, Tasmania.

Sala, O., F. Chapin, J. Armesto, E. Berlow, J. Bloomfield, R. Dirzo, E. Huber-Sanwald, L. Huenneke, R. Jackson, A. Kinsig, R. Leemans, D. Lodge, H. Mooney, M. Oesterheld, N. Poff, M. Sykes, B. Walker, M. Walker \& D. Wall. 2000. Global biodiversity scenarios for the year 2100. Science 287: 1770-1774.

SAX, D.F. 2002. Native and naturalized plant diversity are positively correlated in scrub communites of California and Chile. Diversity and Distributions 8: 193-210. 
Seipel, T., C. Kueffer, L.J. Rew, C.C. Daehler, A. Pauchard, B.J. Naylor, J.M. Alexander, P.J. Edwards, C.G. Parks, J.R. Arévalo, L.A. Cavieres, H. Dietz, G. Jakobs, K. McDougall, R. Оtтo \& N. Walsh. 2012. Processes at multiple scales affect richness and similarity of non-native plant species in mountains around the world. Global Ecology and Biogeography 21: 236-246.

Shea, K. \& P. Chesson. 2002. Community ecology theory as a framework for biological invasions. Ecology \& Evolution 17(4): 170-176.

Sher, A.A. \& L.A. Hyatt. 1999. The disturbed resource-flux invasion matrix: a new frameworkfor patterns of plant invasion. Biological. Invasion 1: 107-114.

Stohlgren, T.J., D. Binkley, G.W. Chong, M.A. Kalkhan, L.D. Schell, K.A. Bull, Y. Otsuki, G. Newman, M. Bashkin \& Y. Son. 1999. Exotic plant species invade hot spots of native plant diversity. Ecological Monographs 69: 25-46.

Tilman, D. 1997. Community invasibility, recruitment limitation, and grassland biodiversity. Ecology 78: 81-92.
TrombulaK, S.C. \& C.A. Frissell. 2000. Review of ecological effects of roads on terrestrial and aquatic communities. Conservation Biology 14(1): 18-30.

VidAL,O. 2005. Flora exótica adyacente a senderos remotos en el Parque Nacional Torres del Paine (Magallanes-Chile). Tesis. Facultad de Ciencias. Universidad Austral de Chile. Valdivia, Chile.i-xii, 118 pp. + anexo.

Vitousek, P.M., C.M. D’Antonio, L. Loope, M. Rejmanek \& R. Westbrooks. 1997. Introduced species: a significant component of human-caused global change. New Zealand Journal of Ecology 21: 1-16.

Yates, E.D., D.F. Levia \& C.L. Williams. 2004. Recruitment of three non-native invasive plants into a fragmented forest in southern Illinois. Forest Ecology and Management 190: 119-130.

Wilson, J.R., D.M. Richardson, M. Rouget, S. Proches, M.A. Amis, L. Henderson \& W. Thuiller. 2007. Residence time and potential range: crucial considerations in modelling plant invasions. Diversity Distribution 13: 11-22.

Anexo 1: Tabla con los valores de WRA de las especies introducidas encontradas dentro de las parcelas y el promedio de los porcentajes de cobertura por tipo de sitio (A y B) y del total. En negrita las especies que presentaron mayores valores de WRA (sobre el promedio que fue 29) y los guiones (---) corresponden a las especies que no estaban presentes en un determinado sitio.

ANNEX 1: Table with WRA values of introduced species found within plots and average of percentage coverage by type of site (A and B) and total. In bold letters the species with higher WRA values (over average was 29) and hyphens (---) correspond to species that were not present in a given site.

\begin{tabular}{|c|c|c|c|c|}
\hline \multirow[b]{2}{*}{ EsPECIE } & \multirow[b]{2}{*}{ WRA } & \multicolumn{3}{|c|}{ Promedio del porcentaJe de cobertura (\%) } \\
\hline & & Sitıo A & Siтio B & TOTAL \\
\hline Aira caryophyllea $\mathrm{L}$. & 19 & 0.27 & 0.01 & 0.13 \\
\hline Anagallis arvensis L. & 27 & 1.42 & --- & 0.63 \\
\hline Anthoxanthum odoratum $\mathrm{L}$. & 31 & 10.10 & 1.12 & 5.01 \\
\hline Arrhenatherum elatius (L.) P. Beauv. ex J. Presl \& C. Presl & 29 & 1.30 & - & 0.57 \\
\hline Bromus hordeaceus L. & 27 & 0.87 & 0.10 & 0.43 \\
\hline Conium maculatum $\mathrm{L}$. & 36 & --- & 0.06 & 0.03 \\
\hline Dactylis glomerata L. & 27 & 1.48 & 0.06 & 0.68 \\
\hline Digitalis purpurea L. & 15 & --- & 0.03 & 0.01 \\
\hline Holcus lanatus L. & 32 & 14.18 & 0.09 & 6.30 \\
\hline Hypochaeris radicata $\mathrm{L}$. & 29 & 2.22 & 0.18 & 1.07 \\
\hline Leucanthemum vulgare Lam. & 25 & 0.07 & --- & 0.03 \\
\hline Lotus pedunculatus Cav. & 31 & 4.28 & 0.44 & 2.11 \\
\hline Plantago lanceolata $\mathrm{L}$. & 29 & 1.78 & 0.34 & 0.96 \\
\hline Prunella vulgaris L. & 34 & 2.52 & 9.65 & 6.19 \\
\hline Ranunculus repens $\mathrm{L}$. & 36 & 17.13 & 14.12 & 14.87 \\
\hline Rosa rubiginosa $\mathrm{L}$. & 35 & --- & 0.01 & 0.01 \\
\hline Rubus constrictus P.J. Müll. \& Lefèvre & 36 & 1.61 & 1.12 & 1.27 \\
\hline Rumex acetosella $\mathrm{L}$. & 33 & 0.08 & 0.12 & 0.10 \\
\hline Rumex obtusifolius L. & 35 & 0.73 & --- & 0.32 \\
\hline Solanum nigrum L. & 25 & --- & 0.03 & 0.01 \\
\hline Taraxacum officinale G. Weber ex F.H. Wigg. & 31 & 0.08 & 0.04 & 0.06 \\
\hline Trifolium pratense & 17 & --- & 0.09 & 0.04 \\
\hline Trifolium repens $\mathrm{L}$. & 30 & 0.08 & $\mathbf{0 . 0 3}$ & 0.07 \\
\hline Veronica serpyllifolia $\mathrm{L}$. & 14 & 0.05 & 0.10 & 0.05 \\
\hline
\end{tabular}

Recibido: 26.09.14

Aceptado: 05.05.15 